\title{
Adenovirus-mediated ING4/IL-24 double tumor suppressor gene co-transfer enhances antitumor activity in human breast cancer cells
}

\author{
YAODONG ZHAO $^{1,2^{*}}$, ZHENGYI LI $^{2 *}$, WEIHUA SHENG ${ }^{2}$, JINGCHEN MIAO $^{2}$ and JICHENG YANG ${ }^{2}$ \\ ${ }^{1}$ Shanghai Tenth People's Hospital, Tongji University School of Medicine, Shanghai 200072; \\ ${ }^{2}$ Cell and Molecular Biology Institute, College of Medicine, Soochow University, Suzhou 215123, P.R. China
}

Received June 7, 2012; Accepted July 4, 2012

DOI: 10.3892/or.2012.1930

\begin{abstract}
Cancer gene therapy represents a new and promising therapeutic modality for various types of cancer. Two or more anti-oncogenes carried by a single vector could theoretically improve treatment efficacy, reduce side-effects from vectors, and have a satisfactory clinical application prospect; however, this has seldom been studied in breast cancer. The inhibitor of growth 4 (ING4), as a member of the inhibitor of growth tumor suppressor family has potent inhibitory effects on a variety of tumors. Interleukin-24 (IL-24) has also shown broad spectrum and tumor-specific antitumor activities. In this study, we aimed to prove the enhanced antitumor activity of adenovirus-mediated ING4/IL-24 double tumor suppressor gene co-transfer in human breast cancer cells. We assessed the combined effect of the ING4/IL-24 bicistronic adenovirus (Ad-ING4-IL-24) in vitro and in vivo on MDA-MB-231 human breast cancer cells by detecting and comparing the apoptotic status in the bicistronic anti-oncogene group (Ad-ING4-IL-24) and in the ING4 or IL-24 single anti-oncogene groups, and also investigated the possible underlying mechanism. Our results showed that the bicistronic adenovirus-mediated ING4 and IL-24 co-expression induced additive growth suppression and apoptosis as well as an overlapping effect on the upregulation of p21, p27 and Bax, and the downregulation of Bcl-2 and
\end{abstract}

Correspondence to: Professor Jicheng Yang, Cell and Molecular Biology Institute, College of Medicine, Soochow University, No. 199 Renai Road, Suzhou 215123, P.R. China

E-mail: jcyang@suda.edu.cn

*Contributed equally

Abbreviations: Ad, adenovirus; GTU, gene transfer unit; IL-24, interleukin 24; ING4, inhibitor of growth 4; IOD, integral optical density; MOI, multiplicity of infection; MVD, microvessel density; OD, optical density; PBS, phosphate-buffered saline; RT, reverse transcription; VEGF, vascular endothelial growth factor

Key words: antitumor activity, breast cancer, inhibitor of growth 4, interleukin 24, double tumor suppressors survivin in MDA-MB-231 human breast cancer cells in vitro or in vivo. Moreover, Ad-ING4-IL-24 treatment additively reduced $\mathrm{CD} 34$ expression and the microvessel density in MDA-MB-231 xenografted tumors in athymic nude mice, which correlated with the decreased expression of the vascular endothelial growth factor. The enhanced antitumor activity on breast cancer elicited by Ad-ING4-IL-24 was closely associated with the activation of the apoptotic pathways and the additive inhibition of tumor angiogenesis.

\section{Introduction}

Breast cancer is a common malignancy among women and is a leading cause of cancer-related mortality, ranking 2nd after lung cancer (1). The incidence of breast cancer has drastically increased over the past several decades. The treatment of breast cancer includes surgery, radiation and drugs (hormone therapy and chemotherapy). Unfortunately, the poor 5-year survival rate of patients with breast cancer has not been improved by current treatments, primarily due to the propensity of breast cancer cells to metastasize as well as their resistance to chemotherapy and radiation. Thus, there is an increased interest in finding new agents for breast cancer treatment. Cancer gene therapy represents a new and promising therapeutic modality for cancer. It was evaluated as one of the top 10 scientific discoveries in 2009 by Science (2). Adenovirus (Ad) is one of the most promising vectors for cancer gene therapy. Adenoviral vectors harboring therapeutic genes have been used successfully for gene transfer in vitro and in vivo.

ING4, a member of the inhibitor of growth (ING) family, may significantly induce tumor growth suppression via the induction of cell cycle alteration, apoptosis and inhibition of tumor angiogenesis (3-8). In a previous study, we demonstrated that the Ad-mediated ING4 gene remarkably inhibited the growth of MDA-MB-231 breast tumors in vitro and in vivo (9). However, cancer is characterized by a multistep process of genetic and molecular changes in oncogenes and tumor suppressor genes, which limits the efficacy of a single genemediated cancer therapy due to the difficulty in finding a pivotal gene conferring its occurrence and progression. Therefore, a multigene-based combination therapy may prove to be effective in breast cancer gene therapy. 
The other tumor suppressor, interleukin-24 (IL-24), originally identified in human melanoma cells treated with interferon- $\beta$ and mezerein by subtraction hybridization, is a unique cytokine-tumor suppressor belonging to the IL-10 family $(10,11)$. Extensive studies have shown that IL-24 displays ubiquitous antitumor properties and tumor-specific killing activity in a broad spectrum of cancer cells but not in normal cells (12). IL-24 may also inhibit tumor angiogenesis by directly suppressing vascular endothelial cell differentiation and migration $(13,14)$, indirectly downregulating the production of pro-angiogenic factors $(13,15,16)$ and by repressing tumor cell invasion and migration via the downregulation of the phosphatidylinositol 3-kinase, focal adhesion kinase and matrix metalloproteinase-2 (17). Thus, ING4 and IL-24, as promising tumor suppressors negatively modulate tumor growth via multiple pathways.

At present, there are two main methods for multigene therapy: the first one involves the target cells being transfected or infected with multiple independent vectors carrying different genes simultaneously, with the advantage of conveniently adjusting the proportion of each expression-vector combination and co-ordination of time and the disadvantage of the efficiency of multigene co-expression being low and onerous $(18,19)$. The second method involves the co-expression of multiple genes in one identical vector (20). Compared with a number of independent vectors carrying different genes to achieve co-expression, a multigene co-expression vector may increase the efficiency of transfection and expression. Since the low efficiency of gene transfer is the bottleneck in gene therapy, we constructed the ING4/IL-24 bicistronic Ad-mediated gene co-transfection vector (Ad-ING4-IL-24) (21).

The therapeutic potential of the conjunction of ING4 and IL-24 for cancer has not been reported in breast cancer. To enhance the therapeutic efficacy and develop a novel combination therapeutic modality for breast cancer, based on the antitumor features of ING4 and IL-24, we hypothesized that the combination treatment with ING4 and IL-24 tumor suppressors would elicit an enhanced antitumor efficacy. Therefore, in this study, we investigated the potential combined effect of the ING4 and IL-24 double tumor suppressor genes (Ad-ING4-IL-24) against MDA-MB-231 human breast cancer cells in vitro and in vivo in an athymic nude mouse model and elucidated its underlying molecular mechanism.

\section{Materials and methods}

Vectors, cell lines, reagents and mice. The Ad-ING4-IL-24 and Ad replication-incompetent adenoviral vectors were constructed and maintained in our laboratory (21). The MDA-MB-231 human breast cancer cell line was purchased from the American Type Culture Collection (Shanghai, China) and cultured in RPMI-1640 (Gibco, Shanghai, China) supplemented with 10\% fetal bovine serum (HyClone, Shanghai, China). The reverse transcription (RT)-PCR detection kit was purchased from Invitrogen (Shanghai, China). The MTT kit was purchased from Sigma (Shanghai, China). The Annexin V-PE/7-AAD apoptosis detection kit was purchased from BD Biosciences (Shanghai, China). The In Situ Cell Death Detection kit was purchased from Roche Applied Science (Shanghai, China). The polyclonal anti-ING4 antibody was purchased from Abcam (Shanghai,
China). The monoclonal anti-IL-24 antibody was purchased from R\&D Systems (Shanghai, China). The antibodies specific for $\mathrm{p} 21, \mathrm{p} 27, \mathrm{Bcl}-2, \mathrm{Bax}$, survivin and $\beta$-actin were purchased from Cell Signaling (Shanghai, China). The Ultrasensitive ${ }^{\mathrm{TM}}$ SP kit was purchased from Fuzhou Maixin (Fuzhou, China). The athymic nude mice were purchased from the Shanghai Experimental Animal Center (Shanghai, China) and maintained in the animal facility at Soochow University with the approval of the Animal Research Ethics Committee of Soochow University. The human IL-24 enzyme-linked immunosorbent assay (ELISA) kit was purchased from Boster (Wuhan, China).

Reverse transcription (RT)-PCR analysis. The apoptosisrelated genes, Bax, Bcl-2 and survivin, in MDA-MB-231 human breast cancer cells were determined by RT-PCR analysis. Briefly, the MDA-MB-231 cells $\left(5 \times 10^{6}\right)$ were infected with Ad-ING4, Ad-IL-24, Ad-ING4-IL-24 or Ad used as a blank control at a multiplicity of infection (MOI) of 100 as previously described (5), or without an Ad (phosphate-buffered saline, PBS) control. After $48 \mathrm{~h}$ of treatment, the infected and uninfected MDA-MB-231 tumor cells were collected, and total cellular RNA was extracted with TRIzol for RT-PCR. The PCR was carried out using cDNA as templates and primers as following: 5'-GGA TGC GTC CAC CAA GAA-3' and 5'-GCA CTC CCG CCA CAA AGA-3' for Bax; 5'-TGT GGC CTT CTT TGA GTT CG-3' and 5'-CTA CCC AGC CTC CGT TAT CC-3' for Bcl-2; 5'-GCA TGG GTG CCC CGA CGT TG-3' and 5'-GCT CCG GCC AGA GGC TCA A-3' for survivin; 5'-TGA TGA CAT CAA GAA GGT GGT GAA-3' and 5'-TCC TTG GAG GCC ATG TGG GCC-3' for human GAPDH. The RT-PCR products were then analyzed by $1 \%$ agarose gel electrophoresis.

Detection of cytotoxicity by crystal violet staining. The MDA-MB-231 human breast cancer cells were planted in a 24-well plate and $24 \mathrm{~h}$ later cells were infected with $\mathrm{Ad}$, Ad-ING4, Ad-IL-24 and Ad-ING4-IL-24, respectively at various MOIs $(0,10,25,50,100$ and 200). After a continuous incubation for 4 days at $37^{\circ} \mathrm{C}$, the medium was removed and $500 \mu \mathrm{l}$ crystal violet solutions ( $2 \%$ crystal violet in $20 \%$ methanol) were added to each well for staining; and 15 min later all wells were washed and images were captured.

MTT assay. The in vitro cytotoxic effect of Ad-ING4-IL-24 on MDA-MB-231 human breast cancer cells was evaluated by MTT assay. Briefly, the MDA-MB-231 tumor cells were dispensed into 96-well culture plates at $1 \times 10^{4}$ cells/well. After a 24-h incubation at $37^{\circ} \mathrm{C}$, the MDA-MB-231 tumor cells were infected with Ad-ING4, Ad-IL-24, Ad-ING4-IL-24 or Ad, used as a blank control at $100 \mathrm{MOI}$ or without Ad (PBS control) and cultured for the indicated time periods (0-4 days). Before treatment and at different time-points after treatment, the viability of the MDA-MB-231 tumor cells was analyzed using an MTT kit according to the manufacturer's instructions. Inhibition ratios were calculated using the following formula: inhibition ratio $=\left[\right.$ optical density $(\mathrm{OD})_{570}$ of control group - $\mathrm{OD}_{570}$ of experimental group $] / \mathrm{OD}_{570}$ of control group.

Flow cytometric analysis of apoptosis. The MDA-MB-231 human breast cancer cells $\left(1 \times 10^{6}\right)$ were cultured with Ad-ING4, Ad-IL-24, Ad-ING4-IL-24 or Ad at 100 MOI or without an 
Ad (PBS control), respectively. After $48 \mathrm{~h}$, the treated and untreated MDA-MB-231 tumor cells were harvested, washed in cold PBS and apoptosis was assessed by flow cytometric analysis using the Annexin V-PE/7-AAD apoptosis detection kit following the manufacturer's instructions. Briefly, the treated and untreated MDA-MB-231 tumor cells $\left(1 \times 10^{6}\right)$ were incubated with $5 \mu \mathrm{l}$ Annexin V-PE (early apoptotic marker) and $5 \mu 1$ 7-AAD (late apoptotic marker) in $100 \mu \mathrm{l}$ of $1 \mathrm{X}$ Annexin V binding buffer at room temperature. After a 15-min incubation, $400 \mu \mathrm{l}$ of $1 \mathrm{X}$ binding buffer were added and the apoptotic cells were then analyzed by flow cytometry. Another section of the cells in each group were also fixed with cold $70 \%$ ethanol for $12 \mathrm{~h}$ and then the cell cycles in each group were detected by flow cytometry after propidium iodide (PI) staining.

ELISA analysis. The Ad-mediated secretory expression of vascular endothelial growth factor (VEGF) in MDA-MB-231 human breast cancer cells was detected by ELISA analysis. Briefly, the MDA-MB-231 tumor cells $\left(5 \times 10^{6}\right)$ were infected with Ad-ING4, Ad-IL-24, Ad-ING4-IL-24 or Ad used as a blank control at $100 \mathrm{MOI}$ or without an Ad (PBS control) in a 10-ml medium, respectively. After $24 \mathrm{~h}$ of treatment, the cellular culture supernatants generated from Ad-ING4, Ad-IL-24, Ad-ING4-IL-24 or Ad-infected and uninfected MDA-MB-231 tumor cells were harvested, and the amount of VEGF in the culture supernatants was analyzed by ELISA using a human VEGF ELISA kit according to the manufacturer's instructions.

Animal studies. The athymic nude mice (5/group) were subcutaneously (s.c.) inoculated on their armpits of the right anterior limbs with $2 \times 10^{6}$ MDA-MB-231 human breast cancer cells, and then monitored daily for tumor growth. Tumor volume was measured with a caliper and calculated by the formula, tumor size $=a b^{2} / 2$, where ' $a$ ' is the larger and ' $b$ ' is the smaller of the 2 dimensions. When the tumors grew up to a mean tumor volume of $\sim 0.1 \mathrm{~cm}^{3}$, the MDA-MB-231 human breast cancer xenografted tumor-bearing mice were intratumorally (i.t.) injected with PBS (PBS control) or $1 \times 10^{8}$ gene transfer unit (GTU) of Ad-ING4, Ad-IL-24, Ad-ING4-IL-24 and Ad every other day for a total of 6 times, respectively. Tumor progression and regression were monitored and tumor volume was measured daily. In addition, the tumor-bearing mice were sacrificed 12 days after the treatments and the xenografted tumors were removed, weighed, fixed by $10 \%$ neutral formalin and embedded in paraffin for hematoxylin \& eosin (H\&E) staining and immunohistochemical analysis. The tumor inhibition rate was calculated using the formula: inhibition rate $=$ (tumor weight of PBS group - tumor weight of therapy group)/tumor weight of PBS group x100\%.

Immunohistochemistry analysis. The expression of p21, p27, Bcl-2, Bax, survivin, VEGF and CD34 in MDA-MB-231 human breast cancer s.c. xenografted tumors was determined by immunohistochemistry analysis using an Ultrasensitive ${ }^{\mathrm{TM}} \mathrm{SP}$ kit according to the manufacturer's instructions. The presence of buffy or brown diaminobenzidine precipitates is indicative of positive reactivity. The integral optical density (IOD) of immunohistochemical intensity was then calculated using Image-Pro Plus 6.0 software. Microvessel density (MVD),

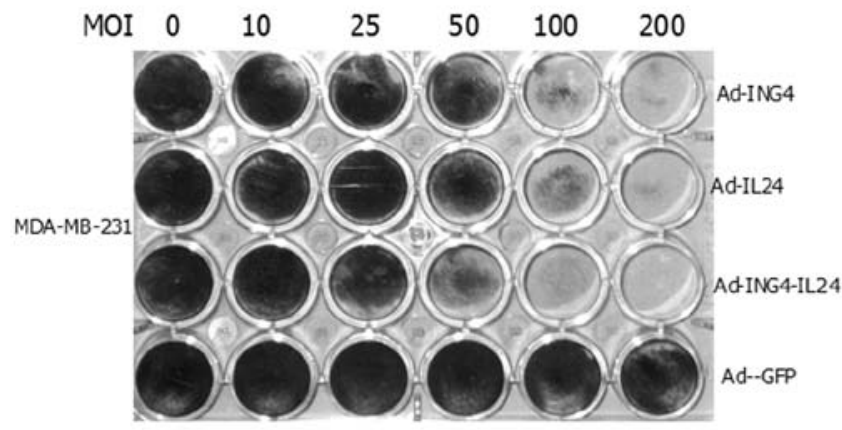

Figure 1. Crystal violet staining in each group.

detected by immunostaining for CD34, was determined as previously described by Weidner (22). Any endothelial cell cluster immunoreactive for CD34 clearly separated from adjacent microvessels was considered as a single countable vessel. Each value represents IOD or microvessels counted at a high-power view (x400) by a microscope. The mean value represents the average number derived from the 5 high-power fields of each case.

Evaluation of combinatorial interaction. The interactive effects of ING4 and IL-24 by Ad-mediated ING4 and IL-24 co-expression were evaluated by calculating the $\mathrm{Q}$-value using the formula (23), $\mathrm{Q}=\mathrm{F}(\mathrm{A}+\mathrm{B}) / \mathrm{FA}+(1-\mathrm{FA}) \mathrm{FB}$, where $\mathrm{F}(\mathrm{A}+\mathrm{B})$ represents the fraction affected by treatment with Ad-ING4-IL-24 compared to the untreated control group, FA represents the fraction affected by Ad-ING4 alone, and FB represents the fraction affected by Ad-IL-24 alone. A value of $Q>1.15$ indicates a synergistic effect between ING4 and IL-24, Q $<0.85$ indicates an antagonistic effect and a vaule of $\mathrm{Q}$ between 0.85 and 1.15 indicates an additive effect.

Statistical analysis. All data are presented as the means \pm standard deviation (SD). The significance of the difference between the groups was evaluated by one-way and two-way repeated measures analysis of variance (ANOVA) and multiple comparisons with SPSS 10.0 software. A value of $\mathrm{P}<0.05$ was considered to indicate a statistically significant difference.

\section{Results}

Cytotoxicityinducedbytumorsuppressorgenes.MDA-MB-231 human breast cancer cells infected with Ad-ING4-IL-24 in the 24-well plate were more slightly stained by crystal violet than the cells infected with Ad-GFP, Ad-ING4 or Ad-IL-24 at a same MOI (Fig. 1). The results indicated that Ad-ING4-IL-24 are more highly toxic to MDA-MB-231 tumor cells than Ad-ING4, Ad-IL-24 or Ad-GFP. Moreover, even at 100 MOI the blank virus vector of Ad-GFP had no obvious cytotoxic effect on MDA-MB-231 tumor cells.

Enhanced tumor suppression by ING4 and IL-24 co-expression. To investigate whether the ING4 plus IL-24 combination treatment elicits enhanced antitumor effects, we co-expressed ING4 and IL-24 double tumor suppressor genes by the ING4/IL-24 bicistronic Ad-mediated gene co-transfer and evaluated the 
A

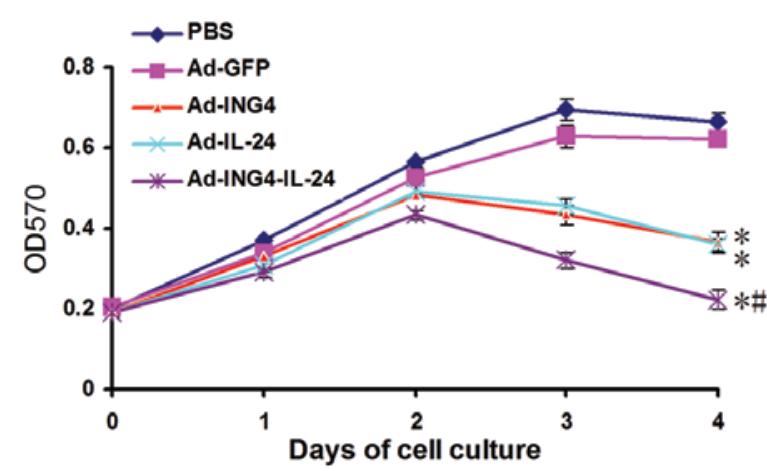

C

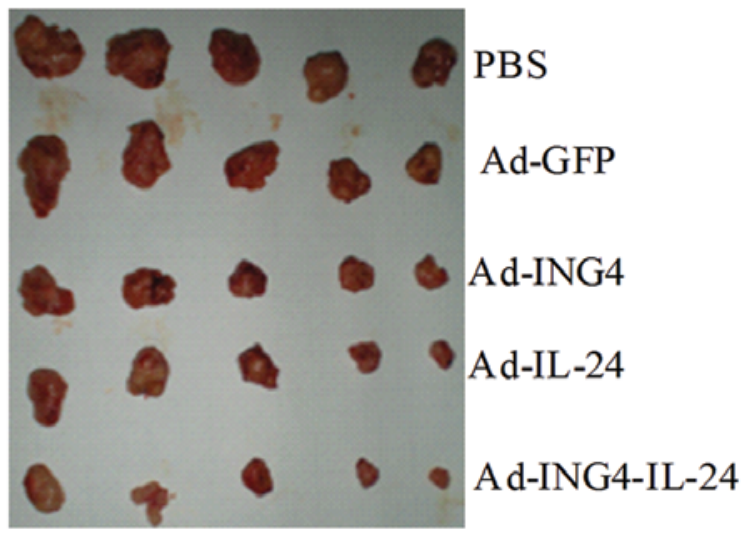

B

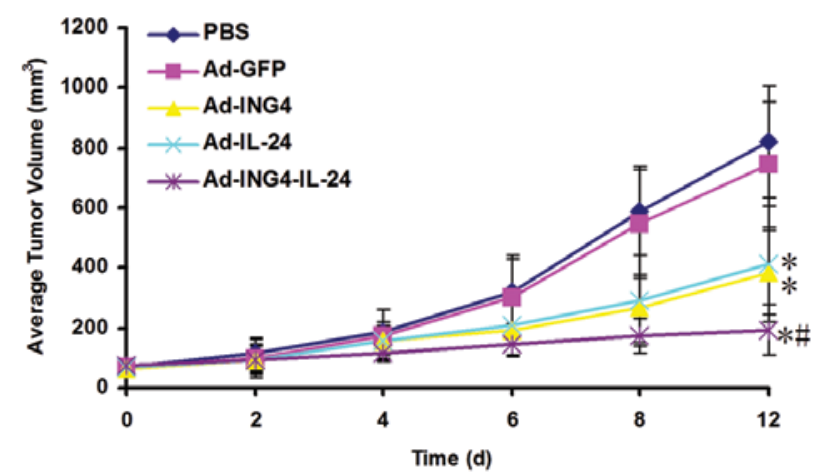

D

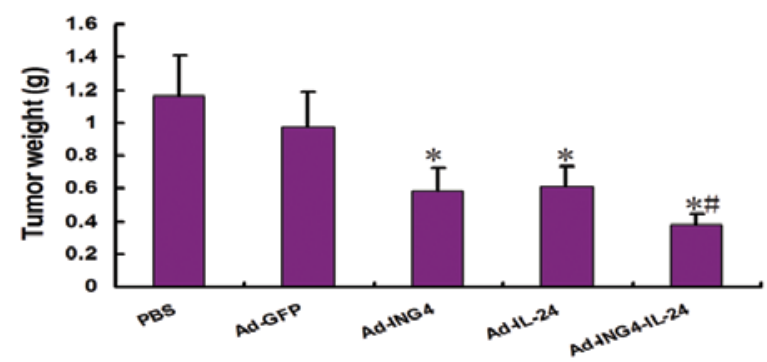

E

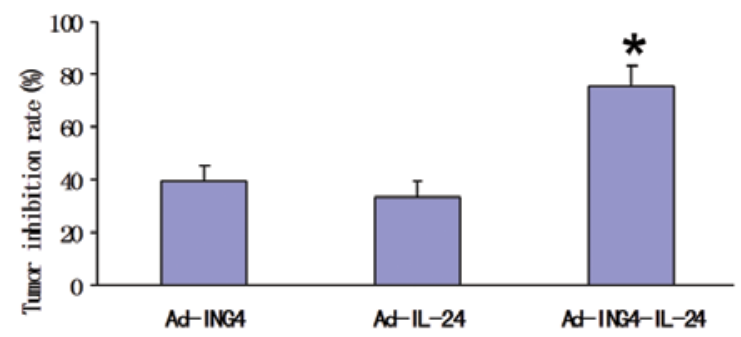

Figure 2. Ad-ING4-IL-24 enhanced tumor suppression in MDA-MB-231 human breast cancer cells. (A) The in vitro cytotoxic effect of Ad-ING4-IL-24 on MDA-MB-231 human breast cancer cells. The MDA-MB-231 human breast cancer cells were treated with Ad-ING4, Ad-IL-24, Ad-ING4-IL-24 or Ad-GFP used as an Ad control at the optimal MOI of 100 or PBS as the control for the indicated time periods ( $0-4$ days), respectively. The survival cells were evaluated at days $0,1,2,3$ and 4 after treatment by MTT assay. ${ }^{*} \mathrm{P}<0.05$ compared with the PBS and Ad-GFP groups; ${ }^{*} \mathrm{P}<0.05$ compared with the Ad-ING4 and Ad-IL-24 groups $(\mathrm{Q}=1.21$ on day 4 after treatment), two-way repeated measures ANOVA and multiple comparisons, $\mathrm{n}=4$ replicates/condition. (B) The MDA-MB-231 human breast cancer xenografted tumor volume before and after treatment. Xenografted tumors were removed at 12 days after treatment and measured. ${ }^{\text {"P }}<0.05$ compared with the PBS and Ad-GFP groups; ${ }^{~} \mathrm{P}<0.05$ compared with the Ad-ING4 and Ad-IL-24 groups, one-way and two-way repeated measures ANOVA and multiple comparisons, $\mathrm{n}=5$ mice/condition. Data shown are representative of 3 independent experiments. (C) Tumor masses removed from each group. (D) The MDA-MB-231 human breast cancer xenografted tumor weight was measured. "P $<0.05$ compared with the PBS and Ad-GFP groups; ${ }^{*} \mathrm{P}<0.05$ compared with the Ad-ING4 and Ad-IL-24 groups. One-way and two-way repeated measures ANOVA and multiple comparisons, $\mathrm{n}=5 \mathrm{mice} / \mathrm{condition}$. Data shown are representative of 3 independent experiments. (E) Tumor inhibition rate of MDA-MB-231 human breast cancer xenografted tumors in each treatment group. " $\mathrm{P}<0.05$ compared with the Ad-ING4 and Ad-IL-24 groups, $\mathrm{Q}=1.29$. One-way and two-way repeated measures ANOVA and multiple comparisons, $\mathrm{n}=5$ mice/condition. Data shown are representative of 3 independent experiments.

combined effect on MDA-MB-231 human breast cancer cells. The MDA-MB-231 tumor cells were infected with Ad-ING4, Ad-IL-24, Ad-ING4-IL-24 or Ad at 100 MOI, respectively. The in vitro cell viability was examined daily for 4 days before and after treatment using MTT assay. As shown in Fig. 2A, compared with the Ad and PBS control group, Ad-mediated ING4 and/or the IL-24 expression significantly suppressed MDA-MB-231 human breast cancer cell growth in vitro in a time-dependent manner with a peak inhibition on day 4 after infection $(\mathrm{P}<0.05)$. Interestingly, the combination treatment of ING4 and IL-24 co-expression induced a more significant and synergistic inhibition on the growth of MDA-MB-231 tumor cells compared with the Ad-ING4- and Ad-IL-24treated group, with inhibition ratios of 32,30 and $62 \%$ on day 4 in the Ad-ING4, Ad-IL-24 and Ad-ING4-IL-24 groups, respectively $(\mathrm{P}<0.05 ; \mathrm{Q}=1.21)$. To further explore whether the combination of ING4 with IL-24 results in enhanced antitumor efficacy in vivo, the athymic nude mice ( 5 mice/group) bearing MDA-MB-231 human breast cancer s.c. xenografted tumors were i.t. injected with Ad-ING4, Ad-IL-24, Ad-ING4-IL-24 or Ad $\left(1 \times 10^{8}\right.$ GTU) every other day for a total of 6 times. The tumor volumes were monitored every other day (Fig. 2B) and xenografted tumors were removed (Fig. 2C) at 12 days after treatment and the tumor weight (Fig. 2D and E) was measured. Compared with the Ad-ING4- and Ad-IL-24-treated group, the growth of MDA-MB-231 human breast cancer xenografted tumors in nude mice was more significantly and synergistically retarded in the Ad-ING4-IL-24-treated group $(\mathrm{P}<0.05$; $\mathrm{Q}_{\text {volume }}=1.29$ ), indicating that Ad-ING4-IL-24 administration may also remarkably suppress in vivo MDA-MB-231 human breast cancer s.c. xenografted tumor growth in an athymic nude mouse model with a synergistic effect. 

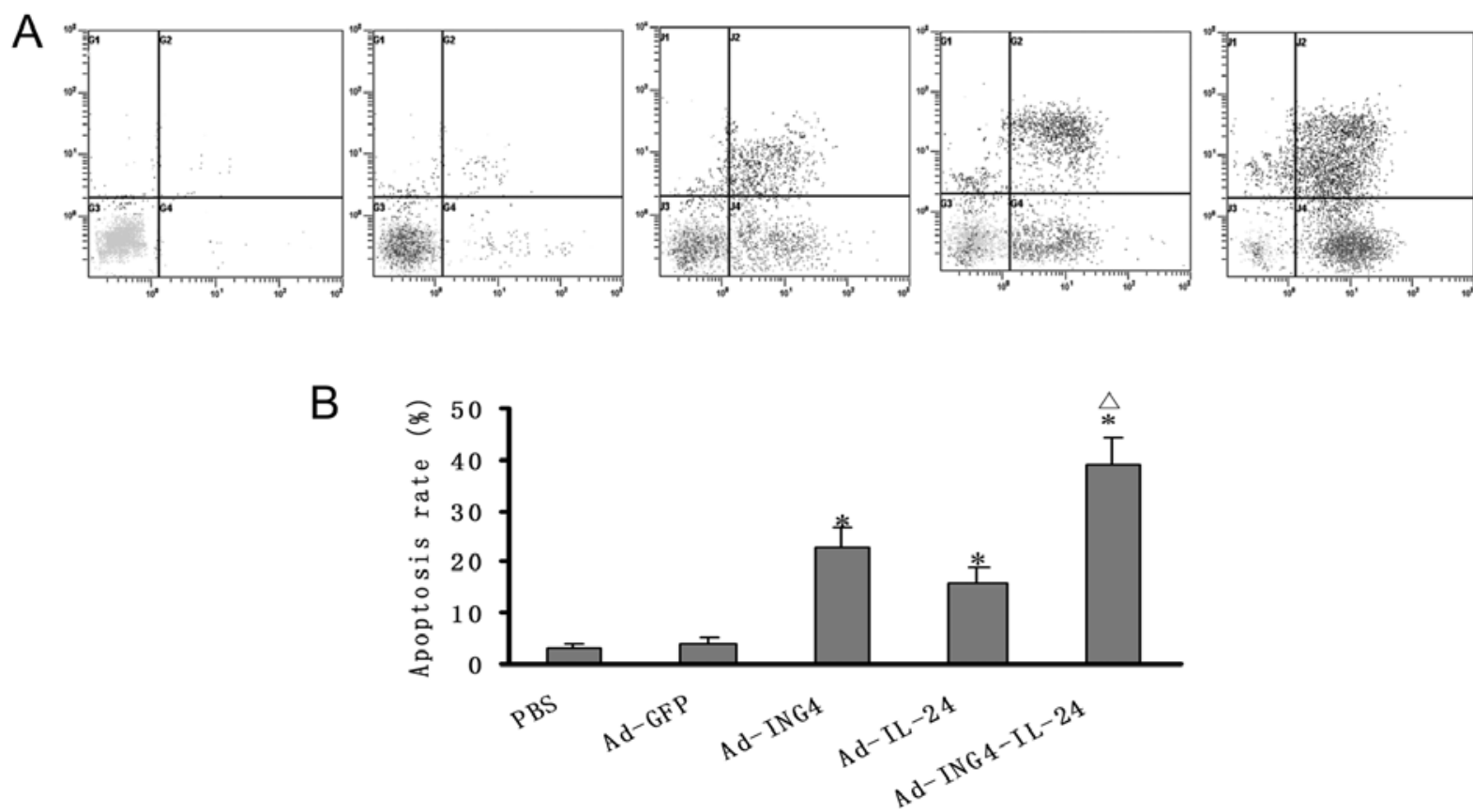

Figure 3. Enhanced induction of apoptosis in MDA-MB-231 human breast cancer cells by Ad-ING4-IL-24. (A) The MDA-MB-231 human breast cancer cells were treated with Ad-ING4, Ad-IL-24, Ad-ING4-IL-24 or Ad-GFP used as the Ad control at the optimal MOI of 100 or PBS as the control for 48 h, and apoptosis was then analyzed using Annexin V-PE/7-AAD double staining by flow cytometry. The Annexin V single-positive cells in the total cell population represented the apoptotic cells. (B) * $\mathrm{P}<0.05$ compared with the PBS and Ad-GFP groups; ${ }^{\triangle} \mathrm{P}<0.05$ compared with the Ad-ING4 and Ad-IL-24 groups $(\mathrm{Q}=1.17)$, one-way repeated measures ANOVA and multiple comparisons, $\mathrm{n}=3$ replicates/condition.

Enhanced apoptosis by ING4 and IL-24 co-expression. To explore the mechanism by which Ad-ING4-IL-24 synergistically inhibits MDA-MB-231 tumor cell growth, the apoptosis of MDA-MB-231 human breast cancer cells treated with Ad-ING4, Ad-IL-24, Ad-ING4-IL-24 or Ad (100 MOI) for $48 \mathrm{~h}$ was analyzed using Annexin V-PE (early apoptotic marker) and 7-AAD (late apoptotic marker) double staining by flow cytometry. As shown in Fig. 3, Ad-ING4-IL-24 treatment resulted in $39.18 \pm 5.08 \%$ of MDA-MB-231 tumor cell apoptosis, whereas there was $2.89,3.92,22.63 \pm 3.16$ and $15.8 \pm 3.12 \%$ of apoptotic MDA-MB-231 tumor cells found in the cells grown in the medium with PBS, Ad, Ad-ING4 and Ad-IL-24, respectively.

Ad-ING4-IL-24 co-operatively regulates apoptotic pathways. In order to further address the underlying molecular mechanism responsible for Ad-ING4-IL-24-promoting apoptosis, the transcriptions and expression levels of the apoptosis-related genes including Bax, Bcl-2, and survivin in Ad-ING4-IL-24-, Ad-ING4-, Ad-IL-24- or Ad-treated and untreated MDA-MB-231 human breast carcinoma cells were analyzed by RT-PCR (in vitro) and immunohistochemistry for tumor tissues. The transcription and expression of Bax in the Ad-ING4, Ad-IL-24 and Ad-ING4-IL-24 groups were significantly increased, and the transcription and expression of $\mathrm{Bcl}-2$ in the Ad-ING4 and Ad-ING4-IL-24 groups were decreased. The transcription and expression of survivin in the Ad-ING4, Ad-IL-24 and Ad-ING4-IL-24 groups were decreased (Fig. 4).

Enhanced cell cycle arrest by ING4 and IL-24 co-expression. To explore the potential mechanism by which Ad-ING4-IL-24 suppresses tumor growth, the cell cycle conditions of the MDA-MB-231 human breast carcinoma cells treated with
PBS, Ad, Ad-ING4, Ad-IL-24 and Ad-ING4-IL-24 for $48 \mathrm{~h}$ were further analyzed using PI staining by flow cytometry. As shown in Fig. 5A and $\mathrm{B}$, the $\mathrm{G} 2 / \mathrm{M}$ phase percentage of MDA-MB-231 tumor cells was $32.36 \pm 3.62 \%$ in group Ad-ING4-IL-24, whereas the percentages were 11.61 \pm 1.26 , $13.52 \pm 1.35,22.52 \pm 3.28$ and $26.24 \pm 2.86 \%$, in the MDA-MB-231 tumor cells grown in medium with PBS, Ad, Ad-ING4 and Ad-IL-24, respectively. To further address the underlying molecular mechanism responsible for Ad-ING4-IL-24 inducing cell cycle arrest, the cell cycle-related proteins, p21 and p27, in the Ad-ING4-IL-24-, Ad-ING4-, Ad-IL-24- or Ad-treated and untreated MDA-MB-231 human breast carcinoma cells were analyzed by immunohistochemistry for tumor tissues. The expression of p21 in the Ad-ING4, Ad-IL-24 and Ad-ING4-IL-24 groups, and the expression levels of p27 in the Ad-IL-24 and Ad-ING4-IL-24 groups were significantly increased (Fig. 5C and D).

Ad-ING4-IL-24 additively reduces tumor angiogenesis. Previous studies have provided evidence that either ING4 or IL-24 may suppress tumor growth by inhibiting angiogenesis $(5,24)$. To examine the combined effect of Ad-mediated ING4 and IL-24 co-expression on tumor angiogenesis, we detected the concentrations of VEGF in the supernatants of Ad-ING4-IL-24-, Ad-ING4-, Ad-IL-24- or Ad-treated and untreated MDA-MB-231 human breast cancer cells by ELISA analysis. The concentrations of VEGF in the Ad-IL-24 and Ad-ING4-IL-24 groups also significantly decreased compared with the PBS and Ad groups (Fig. 6A). The expression levels of VEGF in the Ad-ING4-IL-24, Ad-ING4, Ad-IL-24 or Ad-treated and untreated MDA-MB-231 human breast cancer s.c. xenografted tumors were analyzed by immunohistochemical 
A

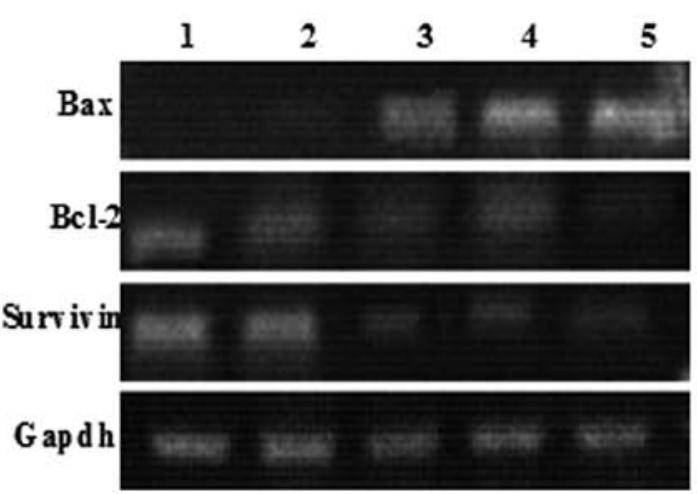

B

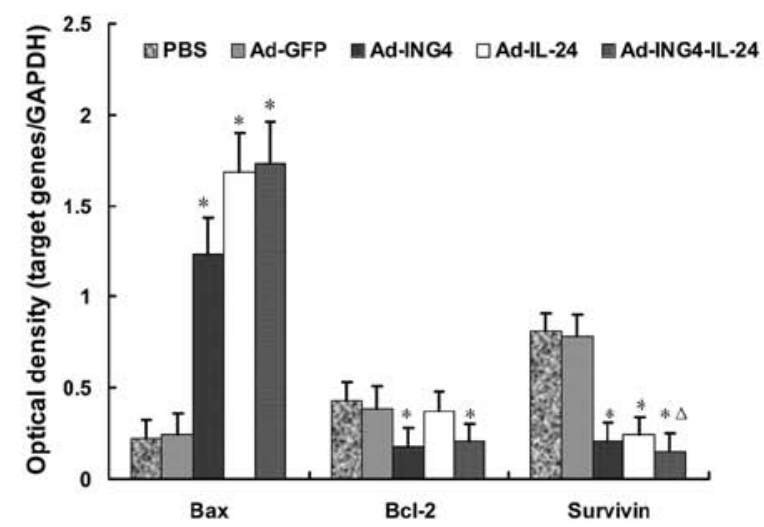

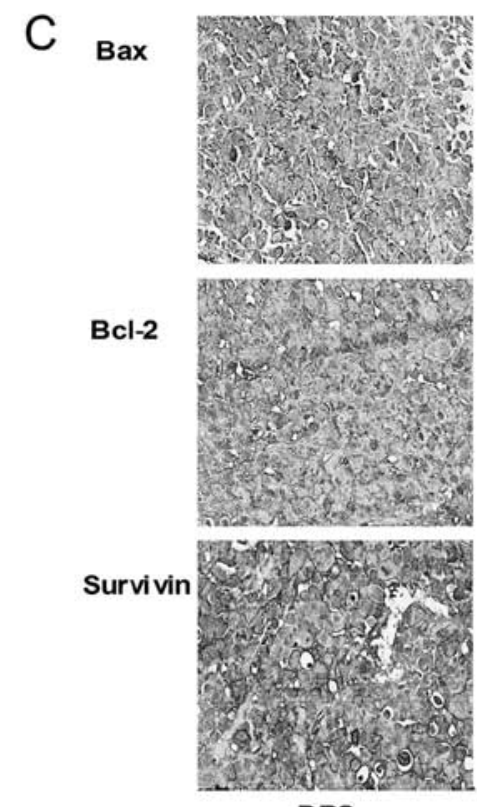

PBS
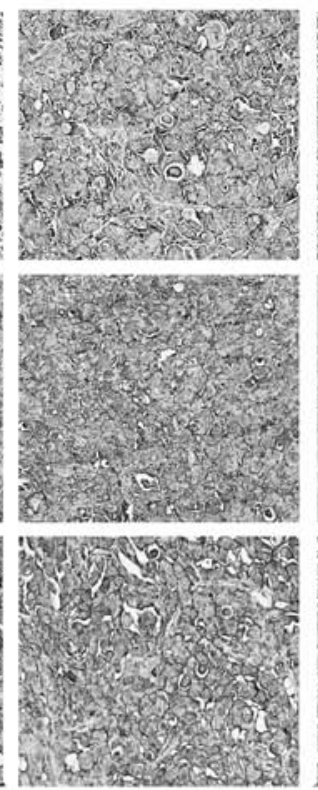

Ad-GFP
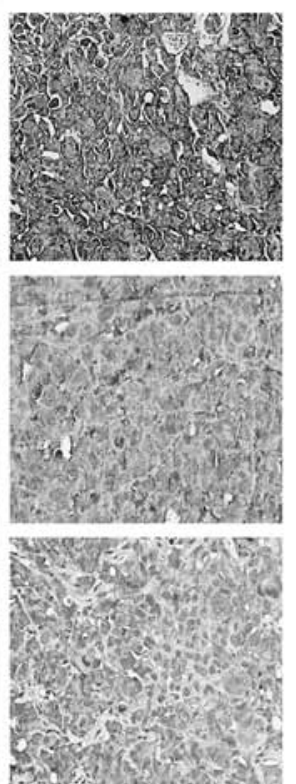

Ad-ING4
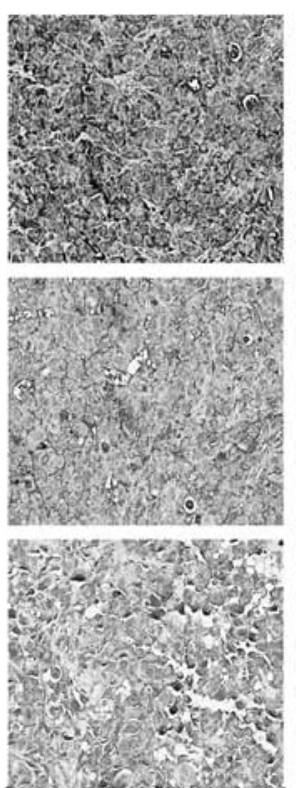

Ad-IL24
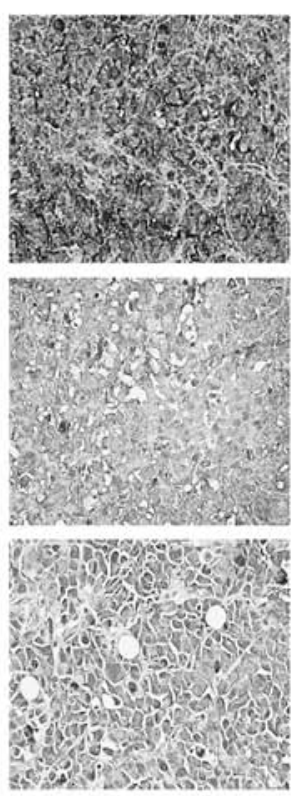

Ad-ING4-IL24

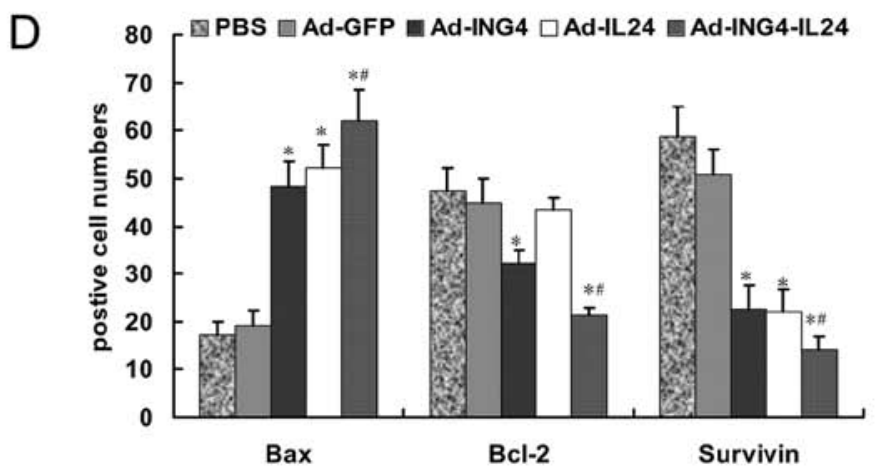

Figure 4. Ad-ING4-IL-24 modulates apoptosis-related molecules. (A) The transcription of Bax, Bcl-2 and survivin in MDA-MB-231 human breast cancer cells was detected by RT-PCR. Lane 1, PBS; lane 2, Ad-GFP; lane 3, Ad-ING4; lane 4, Ad-IL-24; lane 5, Ad-ING4-IL-24. (B) Semiquantitative analysis of these gene transcriptions, " $\mathrm{P}<0.05$ compared with the PBS and Ad-GFP groups; $\triangle \mathrm{P}<0.05$ compared with the Ad-ING4 and Ad-IL-24 groups (Q=1.17), one-way repeated measures ANOVA and multiple comparisons, $\mathrm{n}=3$ replicates/condition. (C) Representative immunohistochemical images for Bax, Bcl-2 and survivin in MDA-MB-231 human breast carcinoma xenografted tumors. The MDA-MB-231 human breast carcinoma s.c. xenografted tumors were maintained in 10\% neutral formalin and embedded in paraffin. Tissue sections were then stained with Bax, Bcl-2 and survivin (ING4 and IL-24 were maintained as the control) by immunohistochemistry using an Ultrasensitive ${ }^{\mathrm{TM}}$ SP kit and examined under a microscope (x400). (D) The IOD of Bax, Bcl-2 and survivin immunohistochemical intensity was quantified by Image-Pro Plus 6.0 software. ${ }^{*} \mathrm{P}<0.05$ compared with the Ad-GFP and PBS groups; ${ }^{*} \mathrm{P}<0.05$ compared with the Ad-ING4 and Ad-IL-24 groups, one-way repeated measures ANOVA and multiple comparisons, $\mathrm{n}=5$ replicates/condition, $\mathrm{n}=5$ observations/representative section.

analysis. The expression levels of VEGF in the Ad-IL-24 and Ad-ING4-IL-24 groups were significantly decreased compared with the PBS and Ad groups (Fig. 6B and D). The MVD in the MDA-MB-231 human breast cancer s.c. xenografted tumors was calculated on the basis of CD34 immunostaining. The CD34-positive staining was mainly presented as brownish yellow or brownish granules in vascular endothelial cells of MDA-MB-231 breast carcinoma xenografted tumors (Fig. 6C). 
A

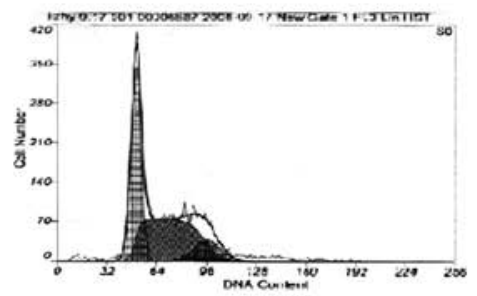

PBS

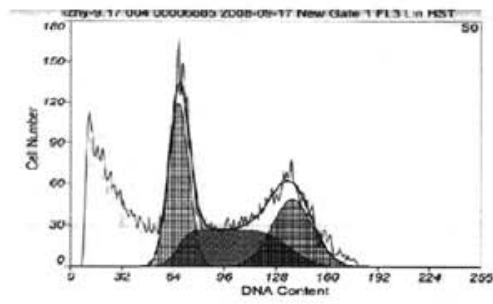

Ad- IL-24

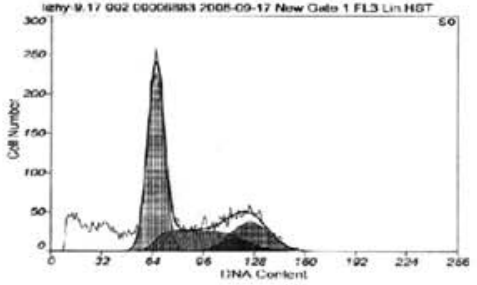

Ad-GFP

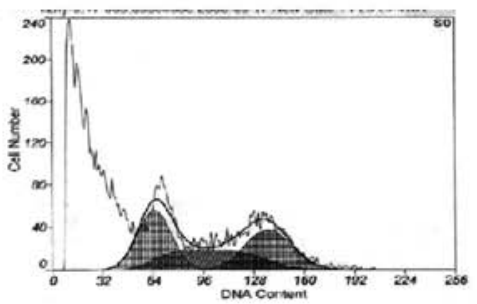

Ad-ING4-IL-24

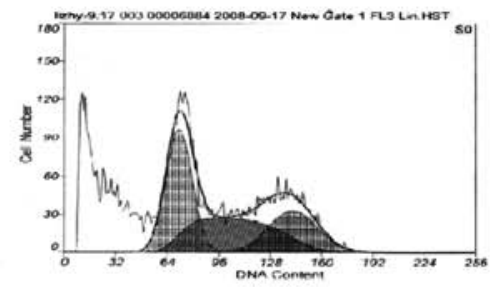

Ad-ING4
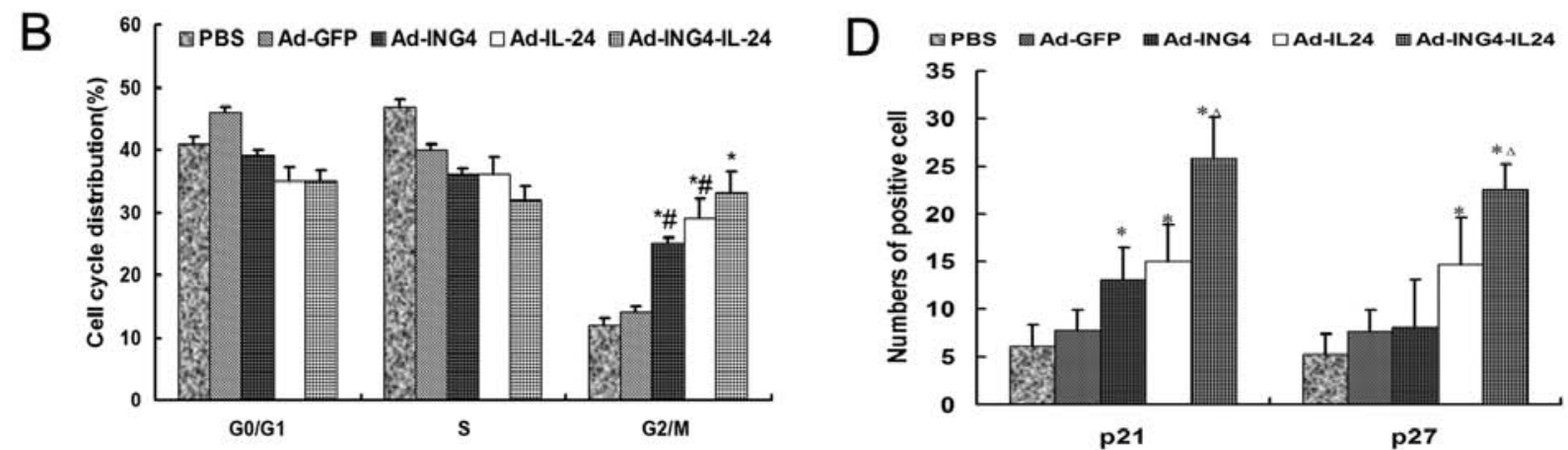

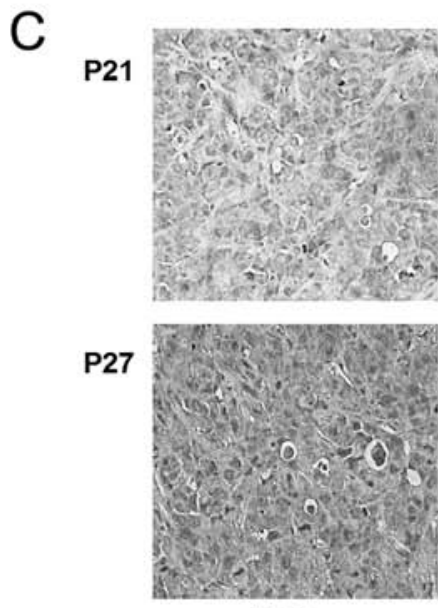

PBS
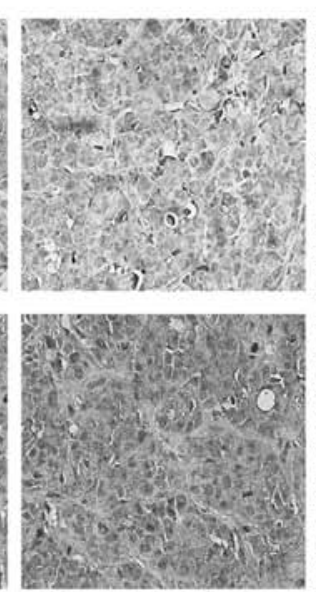

Ad-GFP

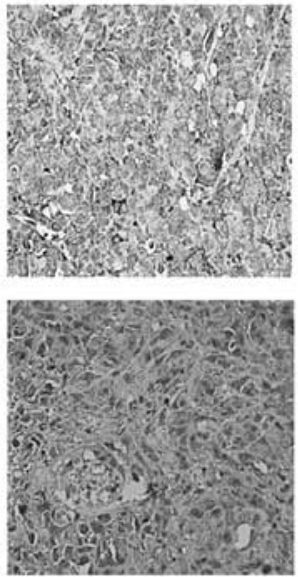

Ad-ING4

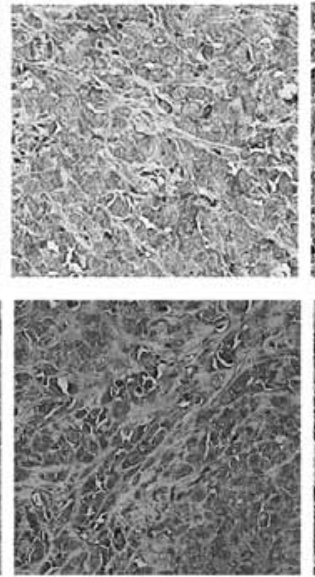

Ad-IL24
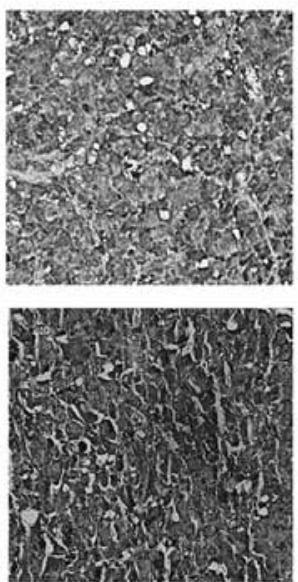

Ad-ING4-IL24

Figure 5. (A) Cell cycle analysis using propidium iodide (PI) staining. (B) Ad-ING4-IL-24 significantly induced G2/M phase arrest in MDA-MB-231 tumor cells ( $\mathrm{P}<0.05$, compared with the Ad-GFP and PBS groups; ${ }^{*} \mathrm{P}<0.05$ compared with Ad-ING4 and Ad-IL-24 groups), by one-way repeated measures ANOVA and multiple comparisons; $\mathrm{n}=3$ replicates/condition. (C) Representative immunohistochemical images for p21 and p27 in MDA-MB-231 human breast carcinoma xenografted tumors. The MDA-MB-231 human breast carcinoma s.c. xenografted tumors were fixed in $10 \%$ neutral formalin and embedded in paraffin. Tissue sections were then stained with $\mathrm{p} 21$ and $\mathrm{p} 27$ by immunohistochemistry using an Ultrasensitive ${ }^{\mathrm{TM}}$ SP kit and examined under a microscope (x400). (D) The IOD of $\mathrm{p} 21$ and p27 immunohistochemical intensity was quantified by Image-Pro Plus 6.0 software. ${ }^{*} \mathrm{P}<0.05$ compared with the Ad-GFP and PBS groups; ${ }^{\wedge} \mathrm{P}<0.05$ compared with the Ad-ING4 and Ad-IL-24 groups, one-way repeated measures ANOVA and multiple comparisons, $\mathrm{n}=5$ replicates/condition, $\mathrm{n}=5$ observations/representative section.

Compared with the PBS and Ad control groups, the CD34 expression of vascular endothelial cells in the Ad-ING4, Ad-IL-24 and Ad-ING4-IL-24 groups was weaker or less (Fig. 6C and D; P<0.05), indicating that the Ad-mediated ING4 and/or IL-24 treatment downregulated the CD34 expression in MDA-MB-231 breast carcinoma xenografted tumor vessels.

\section{Discussion}

Multigene-based combination therapy represents an effective practice in cancer gene therapy, which may achieve greater therapeutic benefits by targeting multiple pathways (25). Recent studies have reported that ING4 as a novel tumor 
A

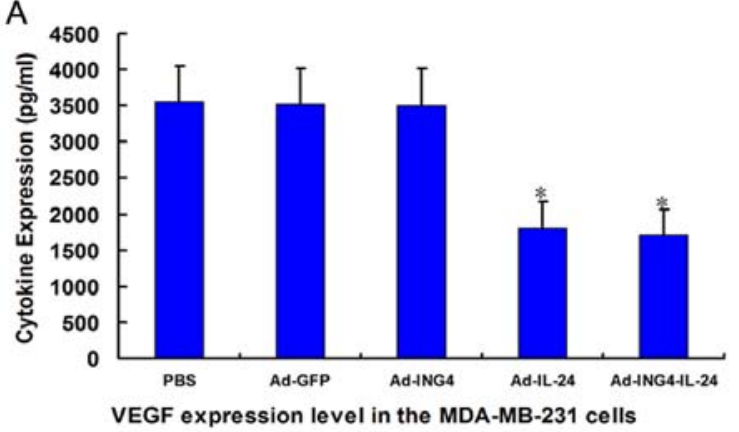

B VEGF

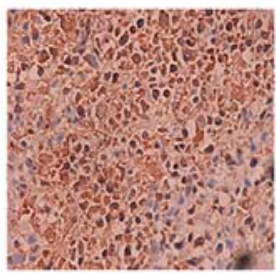

C CD34

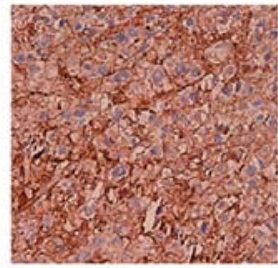

PBS
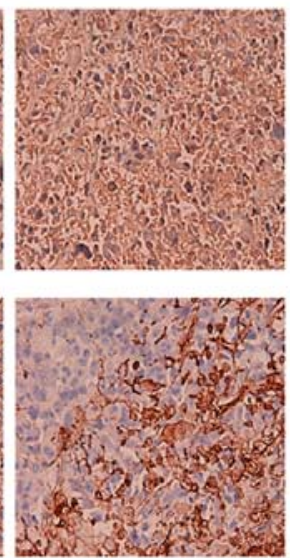

Ad-GFP
D

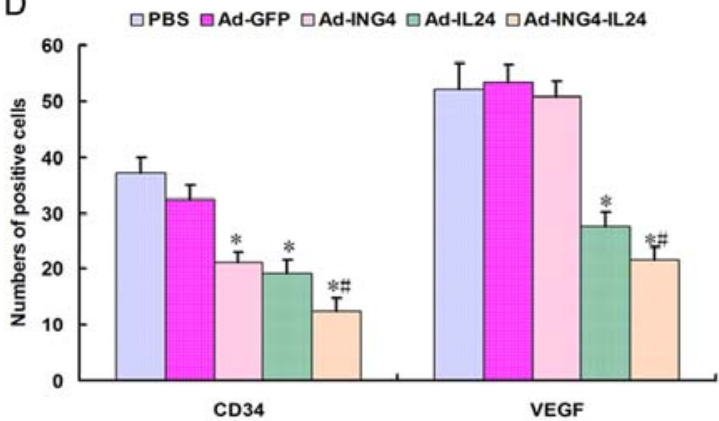

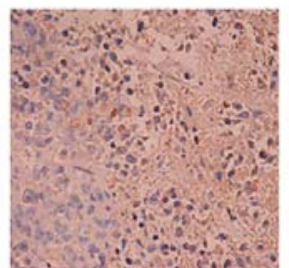
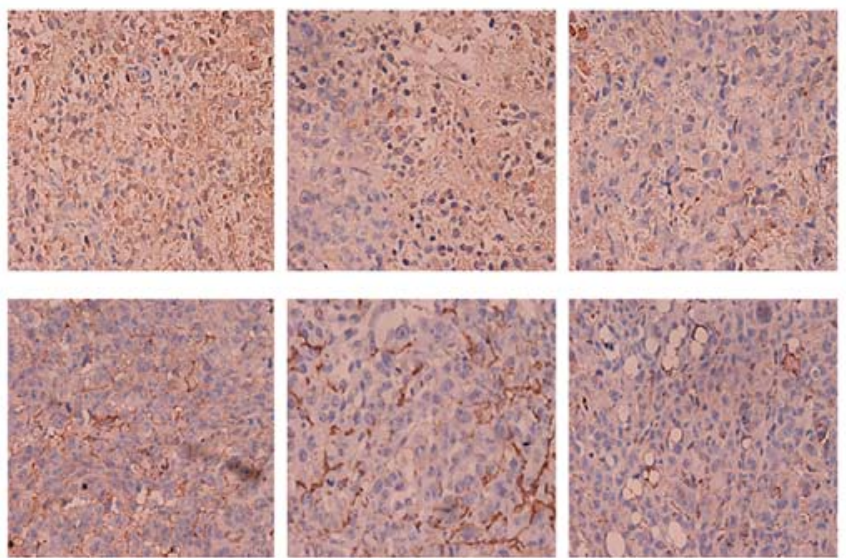

Ad-ING4

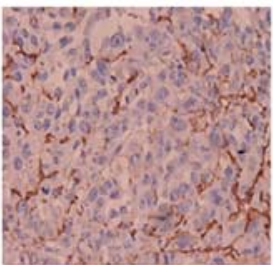

Ad-IL24

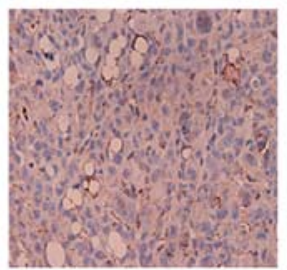

Ad-ING4-IL24

Figure 6. Changes in angiogenesis related cytokine levels before and after treatment. (A) The VEGF concentrations in the supernatants of different groups (B and C) Representative immunohistochemical images for VEGF and CD34 in MDA-MB-231 human breast carcinoma xenografted tumors (x400). (D) The IOD of VEGF and CD34 immunohistochemical intensity quantified by Image-Pro Plus 6.0 software. ${ }^{*}<<0.05$ compared with the Ad-GFP and PBS groups; ${ }^{\#} \mathrm{P}<0.05$ compared with the Ad-ING4 and Ad-IL24 groups. One-way repeated measures ANOVA and multiple comparisons, $\mathrm{n}=5$ replicates/condition, $\mathrm{n}=5$ observations/representative section.

suppressor plays an important role in many cancer-related cellular processes including oncogenesis, cell cycle regulation, apoptosis, DNA damage response, invasion and migration, contact inhibition and tumor angiogenesis, implying that it is a potent tumor suppressor for cancer therapy (8). Subsequent studies have demonstrated that another tumor suppressor, IL-24, as a cytokine-tumor suppressor can discriminate between normal and tumor cells, induce apoptosis, inhibit tumor angiogenesis, stimulate immune responses, promote bystander antitumor activity, and synergize with anticancer drugs and radiation, suggesting that it is also an effective agent for cancer treatment $(10,11)$. However, the therapeutic effect of the combination treatment of ING4 and IL-24 for cancer, remains unreported. Based on the antitumor properties of ING4 and IL-24, we speculated that the combination of ING4 and IL-24 double tumor suppressors would exert enhanced tumor suppression. In this study, we constructed an ING4/ IL-24 biscistronic Ad harboring the ING4 and IL-24 double tumor suppressor genes (Ad-ING4-IL-24) and evaluated its combined therapeutic effect on MDA-MB-231 human breast cancer cells in vitro and MDA-MB-231 human breast cancer s.c xenografted tumors in vivo in an athymic nude mouse model using Ad-mediated ING4 and IL-24 co-transfer. We demonstrated that the combination treatment of Ad-mediated ING4 and IL-24 co-expression induced in vitro synergistic growth suppression and apoptosis in MDA-MB-231 human breast cancer cells. Moreover, Ad-ING4-IL-24 also synergistically inhibited MDA-MB-231 human breast cancer xenografted tumor growth in vivo in athymic nude mice.

Genes regulating apoptosis may be divided into apoptosisrelated oncogenes and anti-oncogenes. The former promote apoptosis (Bax), and the latter are anti-apoptotic (Bcl-2 and survivin). The ratios between Bcl-2/Bax heterodimers and Bax/Bax homodimers appear to be pivotal in deciding the life or death of a cell (26). Bcl-2/Bax constitutes a rheostat that sets the threshold of susceptibility to apoptosis (27). Survivin inhibits apoptosis by interacting with cyclin kinase CDK4, p34, CDC2 and blocking apoptotic signal transduction. Survivin is rarely expressed in normal adult tissues but displays a weak expression in the placenta and thymus (28); however, it is widely expressed in tumors. A high expression of survivin in cancer is closely related to malignant progression, poor prognosis, tumor recurrence and drug resistance (29). p21 and p27, important members of CDK inhibitors belonging to the Cip/Kip family, inhibit cyclin E-CDK2, cyclin A-CDK2, cyclin D-CDK4 and cyclin B1/CDC2 complexes leading to G1 and $\mathrm{G} 2 / \mathrm{M}$ arrest (30-33).

To elucidate the underlying mechanism involved in Ad-ING4-IL-24-mediated synergistic antitumor activity, the in vitro transcription and in vivo expression of apoptosis-related proteins, such as Bcl-2, Bax and survivin in MDA-MB-231 human breast cancer xenografted tumors were assessed by RT-PCR and immunohistochemical analysis. Evidence from in vitro and in vivo experiments demonstrated 
that Ad-mediated ING4 and IL-24 co-expression elicited a co-operative and overlapping effect on the upregulation of the apoptosis promoting gene, Bax, and the downregulation of the anti-apoptotic genes, Bcl-2 and survivin, in MDA-MB-231 human breast cancer cells or tumor tissues. These results may closely account for the Ad-ING4-IL-24-induced synergistic growth inhibition and apoptosis in MDA-MB-231 tumor cells and xenografted tumors.

To uncover other mechanisms of tumor growth inhibition by Ad-ING4-IL-24, the cell cycle conditions of MDA-MB-231 human breast carcinoma cells in different groups were analyzed by flow cytometry. The results showed an obvious G2/M cell cycle arrest in the Ad-ING4 and Ad-IL-24 groups and an overlapping effect in the Ad-ING4-IL-24 group. To explore the potential molecular mechanism, the present study assessed the cell cycle-related molecules, p21 and p27, in MDA-MB-231 human breast cancer s.c. xenografted tumors by immunohistochemical analysis. The results showed that the expression levels of p21 and p27 both significantly increased in the Ad-ING4-IL-24 group, which indicated that the Ad-mediated co-expression of ING4 and IL-24 induced G2/M arrest and inhibited the cell cycle of MDA-MB-231 human breast cancer cells by stimulating the expression of the cell cycle inhibitors, p21 and p27.

In addition, the progressive growth and metastasis of solid tumors are dependent on the process of angiogenesis. It has been shown that ING4 suppresses tumor angiogenesis via the downregulation of IL-8 and osteopontin pro-angiogenic factors by inhibiting the activity of nuclear factor $\kappa \mathrm{B}$ and hypoxia-inducible factor- $1 \alpha(8,24)$. It has also been reported that IL-24 inhibits tumor angiogenesis via directly interacting with the IL-22R1/IL-20R2 heterodimeric receptor in vascular endothelial cells (14) and indirectly reducing pro-angiogenic factor production (13-15).

Animal experimental studies showed that the Ad-mediated ING4 and IL-24 tumor suppressor gene co-transfer reduced tumor volumes significantly, while the tumor inhibition rate of the Ad-ING4-IL-24 group reached 76.5 $\pm 7.4 \%(\mathrm{Q}=1.29)$, demonstrating the synergistic effect of ING4 and IL-24 in vivo. In addition to the promotion of apoptosis and the inhibition of the cell cycle, whether or not the suppression of tumor angiogenesis is also an underlying mechanism involved in the Ad-ING4-IL-24-mediated synergistic antitumor activity, remains unclear. In order to clarify this, we detected the MVD of tumor tissues in each group on the basis of CD34 immunostaining, and the expression levels of VEGF, a key stimulator of vessel formation (34), by ELISA in vitro and immunostaining in vivo. We further found that Ad-ING4-IL-24 additively downregulated CD34 and VEGF expression and decreased MVD in MDA-MB-231 human breast cancer xenografted tumors, which may be another important mechanism involved in the Ad-ING4-IL-24-mediated in vivo enhanced growth inhibition of MDA-MB-231 human breast cancer xenografted tumors in an athymic nude mouse model.

\section{Acknowledgements}

The current research was supported by grants from the National Natural Science Foundation of China (nos. 81001016; 81101909).

\section{References}

1. Jemal A, Siegel R, Ward E, Hao Y, Xu J, Murray T and Thun MJ: Cancer statistics, 2008. CA Cancer J Clin 58: 71-96, 2008.

2. Cartier N, Hacein-Bey-Abina S, Bartholomae CC, et al: Hematopoietic stem cell gene therapy with a lentiviral vector in X-linked adrenoleukodystrophy. Science 326: 818-823, 2009.

3. Shiseki M, Nagashima M, Pedeux RM, et al: p29ING4 and p28ING5 bind to p53 and p300, and enhance p53 activity. Cancer Res 63: 2373-2378, 2003.

4. Zhang X, Xu LS, Wang ZQ, et al: ING4 induces G2/M cell cycle arrest and enhances the chemosensitivity to DNA-damage agents in HepG2 cells. FEBS Lett 570: 7-12, 2004.

5. Xie Y, Zhang H, Sheng W, Xiang J, Ye Z and Yang J: Adenovirusmediated ING4 expression suppresses lung carcinoma cell growth via induction of cell cycle alteration and apoptosis and inhibition of tumor invasion and angiogenesis. Cancer Lett 271: 105-116, 2008.

6. Cai L, Li X, Zheng S, et al: Inhibitor of growth 4 is involved in melanomagenesis and induces growth suppression and apoptosis in melanoma cell line M14. Melanoma Res 19: 1-7, 2009.

7. Xie YF, Sheng W, Xiang J, Zhang H, Ye Z and Yang J: Adenovirus-mediated ING4 expression suppresses pancreatic carcinoma cell growth via induction of cell-cycle alteration, apoptosis, and inhibition of tumor angiogenesis. Cancer Biother Radiopharm 24: 261-269, 2009.

8. Colla S, Tagliaferri S, Morandi F, et al: The new tumor-suppressor gene inhibitor of growth family member 4 (ING4) regulates the production of proangiogenic molecules by myeloma cells and suppresses hypoxia-inducible factor-1 alpha (HIF-1alpha) activity: involvement in myeloma-induced angiogenesis. Blood 110: 4464-4475, 2007

9. Li Z, Xie Y, Sheng W, Miao J, Xiang J and Yang J: Tumorsuppressive effect of adenovirus-mediated inhibitor of growth 4 gene transfer in breast carcinoma cells in vitro and in vivo. Cancer Biother Radiopharm 25: 427-437, 2010.

10. Jiang H, Lin JJ, Su ZZ, Goldstein NI and Fisher PB: Subtraction hybridization identifies a novel melanoma differentiation associated gene, mda-7, modulated during human melanoma differentiation, growth and progression. Oncogene 11: 2477-2486, 1995.

11. Sauane M, Gopalkrishnan RV, Sarkar D, et al: MDA-7/IL-24: novel cancer growth suppressing and apoptosis inducing cytokine. Cytokine Growth Factor Rev 14: 35-51, 2003.

12. Fisher PB: Is mda-7/IL-24 a 'magic bullet' for cancer? Cancer Res 65: 10128-10138, 2005.

13. Saeki T, Mhashilkar A, Swanson X, et al: Inhibition of human lung cancer growth following adenovirus-mediated mda-7 gene expression in vivo. Oncogene 21: 4558-4566, 2002.

14. Ramesh R, Mhashilkar AM, Tanaka F, et al: Melanoma differentiation-associated gene $7 /$ interleukin (IL)-24 is a novel ligand that regulates angiogenesis via the IL-22 receptor. Cancer Res 63: 5105-5113, 2003.

15. Nishikawa T, Ramesh R, Munshi A, Chada S and Meyn RE: Adenovirus-mediated mda-7 (IL-24) gene therapy suppresses angiogenesis and sensitizes NSCLC xenograft tumors to radiation. Mol Ther 9: 818-828, 2004.

16. Inoue S, Branch CD, Gallick GE, Chada S and Ramesh R: Inhibition of Src kinase activity by Ad-mda7 suppresses vascular endothelial growth factor expression in prostate carcinoma cells. Mol Ther 12: 707-715, 2005.

17. Ramesh R, Ito I, Gopalan B, Saito Y, Mhashilkar AM and Chada S: Ectopic production of MDA-7/IL-24 inhibits invasion and migration of human lung cancer cells. Mol Ther 9: 510-518, 2004.

18. Pei Z, Chu L, Zou W, et al: An oncolytic adenoviral vector of Smac increases antitumor activity of TRAIL against HCC in human cells and in mice. Hepatology 39: 1371-1381, 2004.

19. Shen Y, Muramatsu SI, Ikeguchi K, et al: Triple transduction with adeno-associated virus vectors expressing tyrosine hydroxylase, aromatic-L-amino-acid decarboxylase, and GTP cyclohydrolase I for gene therapy of Parkinson's disease. Hum Gene Ther 11: $1509-1519,2000$

20. Ngoi SM, Chien AC and Lee CG: Exploiting internal ribosome entry sites in gene therapy vector design. Curr Gene Ther 4: 15-31, 2004.

21. Sheng WH, Xie YF, Miao JC, et al: The anti-tumor effect by adenovirus-mediated ING4 and IL-24 co-expression on hepatocellular carcinoma in vitro. Chin J Microbiol Immunol 30: 695-703, 2010 
22. Weidner N: Current pathologic methods for measuring intratumoral microvessel density within breast carcinoma and other solid tumors. Breast Cancer Res Treat 36: 169-180, 1995.

23. Wang W, Qin SK, Chen BA and Chen HY: Experimental study on antitumor effect of arsenic trioxide in combination with cisplatin or doxorubicin on hepatocellular carcinoma. World $\mathbf{J}$ Gastroenterol 7: 702-705, 2001.

24. Garkavtsev I, Kozin SV, Chernova O, et al: The candidate tumour suppressor protein ING4 regulates brain tumour growth and angiogenesis. Nature 428: 328-332, 2004.

25. Wilson DR: Viral-mediated gene transfer for cancer treatment. Curr Pharm Biotechnol 3: 151-164, 2002.

26. Meijerink JP, Smetsers TF, Slöetjes AW, Linders EH and Mensink EJ: Bax mutations in cell lines derived from hematological malignances. Leukemia 9: 1828-1832, 1995.

27. Danial NN and Korsmeyer SJ: Cell death: critical control points. Cell 116: 205-219, 2004.

28. Adida C, Crotty PL, McGrath J, Berrebi D, Diebold J and Altieri DC: Developmentally regulated expression of the novel cancer anti-apoptosis gene survivin in human and mouse differentiation. Am J Pathol 152: 43-49, 1998.
29. AItieri DC: The molecular basis and potential role of survivin in cancer diagnosis and therapy. Trends Mol Med 7: 542-547, 2001.

30. Harper JW, Adami GR, Wei N, Keyomarsi K and Elledge SJ: The p21 Cdk-interacting protein Cip1 is a potent inhibitor of G1 cyclin-dependent kinases. Cell 75: 805-816, 1993.

31. Niculescu AB III, Chen X, Smeets M, Hengst L, Prives C and Reed SI: Effects of p21(Cip1/Waf1) at both the G1/S and the $\mathrm{G} 2 / \mathrm{M}$ cell cycle transitions: $\mathrm{pRb}$ is a critical determinant in blocking DNA replication and in preventing endoreduplication. Mol Cell Biol 18: 629-643, 1998.

32. Smits VA, Klompmaker R, Vallenius T, Rijksen G, Mäkela TP and Medema RH: p21 inhibits Thr161 phosphorylation of Cdc2 to enforce the G2 DNA damage checkpoint. J Biol Chem 275: 30638-30643, 2000.

33. Toyoshima $\mathrm{H}$ and Hunter $\mathrm{T}$ : $\mathrm{p} 27$, a novel inhibitor of $\mathrm{G} 1$ cyclinCdk protein kinase activity, is related to p21. Cell 78: 67-74, 1994.

34. Petrova TV, Makinen T and Alitalo K: Signaling via vascular endothelial growth factor receptors. Exp Cell Res 253: 117-130, 1999. 